CORRECTION

https://doi.org/10.1038/s41586-018-0611-3

\title{
Publisher Correction: Evidence that CD32a does not mark the HIV-1 latent reservoir
}

Christa E. Osuna, So-Yon Lim, Jessica L. Kublin, Richard Apps, Elsa Chen, Talia M. Mota, Szu-Han Huang, Yanqin Ren, Nathaniel D. Bachtel, Athe M. Tsibris,

Margaret E. Ackerman, R. Brad Jones, Douglas F. Nixon \& James B. Whitney

Correction to: Nature https://doi.org/10.1038/s41586-018-0495-2, published online 19 September 2018.

In this Brief Communications Arising Comment, owing to a typesetter error, the first three authors (Christa E. Osuna, So-Yon Lim and Jessica L. Kublin) should have been listed as equally contributing authors but were not. This has been corrected online. 